\title{
PERSPECTIVA DE GÉNERO EN PROFESIONALES DE LA SALUD DURANTE LA PANDEMIA DE COVID-19
}

A Gender Approach to Health Professionals During the COVID-19 Pandemic

Ana Rodríguez-Cala ${ }^{1}$, Candela Calle-Rodríguez ${ }^{1}$, Anna Pons-Rodríguez ${ }^{2}$, Noemí DuránGarcía $^{1}$

Autor correspondencia: Ana Rodríguez-Cala

Correo electrónico: arcala@iconcologia.net

1. Instituto Catalán de Oncología. (Hospitalet de Llobregat, España)

2. Institut Català de la Salut. (Lleida, España)

Recibido: 24/07/2020 Aceptado: 27/07/2021

(c) (1) () 


\section{RESUMEN}

INTRODUCCIÓN. La pandemia de COVID-19 ha generado una crisis sanitaria con un contundente impacto social, derivado en gran medida de las políticas de confinamiento de la población. Estudios anteriores han puesto en evidencia las desigualdades de género que generan las pandemias. OBJETIVO. Esta investigación tiene como objetivo identificar el impacto de la pandemia de la COVID-19 en profesionales de la salud desde perspectiva de género, en aspectos de la esfera profesional, familiar y social. MATERIAL Y MÉTODO. El estudio es de tipo exploratorio descriptivo, con una metodología de investigación cuantitativa transversal. El instrumento para la recogida de los datos es un cuestionario estructurado administrado por correo electrónico. Se realiza un análisis descriptivo de las principales características de las personas participantes según el género. RESULTADOS. Los resultados del estudio apuntan a la existencia de desigualdades entre profesionales de la salud, determinadas por el género, en referencia a la participación en la toma de decisiones, la diferente carga que suponen las tareas de cuidado, el impacto en aspectos emocionales y percepciones sobre lo que supone la pandemia para hombres y mujeres. CONCLUSIONES. El papel de la mujer debe ser tenido en cuenta en la gestión de las pandemias. Especialmente debe implicarse a las mujeres en la toma de decisiones en el ámbito de la salud para tratar aspectos relacionados con el abordaje sanitario, así como las consecuencias sociales y emocionales que afectan a las profesionales derivadas de la carga asistencial y la falta de corresponsabilidad en los cuidados.

Palabras clave: género; coronavirus; pandemias; personal de salud; COVID-19.

\section{ABSTRACT}

INTRODUCTION. The COVID-19 pandemic has brought about a health crisis with a strong social impact, largely derived from population confinement policies. Previous studies have highlighted the gender inequalities that pandemics generate. OBJECTIVE. This research aims to identify the impact of the COVID-19 pandemic on health professionals with a gender perspective in aspects related to their work, family, and social spheres. MATERIAL AND METHOD. The study is descriptive exploratory, with a cross-sectional quantitative research methodology. The instrument for data collection is a structured questionnaire administered by email. A descriptive analysis of the main characteristics of the participants according to gender is performed. RESULTS. The results of the study point to the existence of inequalities between health professionals, determined by gender, in reference to participation in decision-making, the different burden of care tasks, the impact on emotional aspects and perceptions about what supposes the pandemic for men and women. CONCLUSIONS. The role of women must be taken into account in the management of pandemics. Women should be especially involved in decision-making in healthcare to deal with the social and emotional consequences that affect health professionals, and which derive from the care burden and the lack of co-responsibility in the provision of care

Keywords: Gender Identity, Coronavirus, Pandemics, Health Personnel, COVID-19. 


\section{INTRODUCCIÓN}

El 11 de marzo de 2020 la Organización Mundial de la Salud (OMS) declara la pandemia por el coronavirus SARS-Cov-2. En España, el mismo mes se declara el estado de alarma en todo el territorio español (Real Decreto 463/2020, de 14 de marzo, por el que se declara el estado de alarma para la gestión de la situación de crisis sanitaria ocasionada por el COVID-19), iniciando un confinamiento de la población que durará, en diferentes grados, hasta el 21 de junio del mismo año.

La pandemia de COVID-19, provocada por el SARS-Cov-2, ha generado un gran impacto en nuestra sociedad, además del sanitario, con consecuencias psicológicas que repercuten en la vida laboral, social y familiar de las personas, según recogen Balluerka et al. (2020). Esta situación afecta especialmente a las mujeres, que sufren mayor precariedad y pobreza laboral en algunos de los sectores afectados por la crisis, como han apuntado Solanas (2020) y Alon et al. (2020).

Además, las mujeres realizan la mayor parte del trabajo doméstico y el cuidado de personas dependientes, como muestra un estudio del Instituto de la Mujer y para la Igualdad de Oportunidades (2020), asumiendo una mayor carga mental. A las dificultades habituales de conciliación y falta de corresponsabilidad, durante el estado de alarma se le suma el cierre de los centros educativos, el teletrabajo y el confinamiento, con la consiguiente sobrecarga de trabajo.

El confinamiento también ha afectado a las mujeres que han hecho teletrabajo y han tenido que compaginarlo con las tareas del hogar y el cuidado de hijos e hijas y personas dependientes. Esta situación ha provocado ansiedad y estrés en muchas personas, especialmente en las mujeres, como evidencia Benlloch (2020).

En el caso de las profesionales de la salud, la pandemia las ha situado en el centro de la crisis sanitaria, por la alta feminización del sector. En España, las mujeres representan el 68\% del personal sanitario (51\% en medicina, $84 \%$ en enfermería, $72 \%$ en farmacia y $82 \%$ en psicología), según los datos publicados por el Instituto de la Mujer y para la Igualdad de Oportunidades (2020) a partir de la Encuesta de Población Activa (EPA).

Así, las mujeres han estado más expuestas a la enfermedad, puesto que su contacto directo con pacientes afectados por coronavirus implica un elevado nivel de exposición al contagio. Situación a la que hay que sumar la presión derivada de doblar turnos y hacer más horas extra. Además de la presión asistencial, se han enfrentado a la situación de 
confinamiento familiar con consecuencias emocionales y psicológicas como observan Liu et al. (2020), y como se ha evidenciado también en anteriores pandemias por Holroyd et al. (2008).

Las proporciones de la crisis de la COVID-19 son aún desconocidas y tendrá graves consecuencias sociales y económicas que afectarán de manera diferente a mujeres y hombres, como apuntan diversos trabajos (véase, por ejemplo, Castellanos-Torres et al., 2020; Wenham et al., 2020; The Lancet, 2020), por lo que la perspectiva de género será crucial para que las soluciones que se implementen eviten que lo peor de la pandemia recaiga sobre ellas.

Con esta investigación se pretende profundizar en algunos de los aspectos que podrían generar desigualdades de género. El objetivo es identificar el impacto de la pandemia de la COVID-19 en profesionales de la salud desde una perspectiva de género, en aspectos de la esfera profesional, familiar y social.

\section{MATERIAL Y MÉTODO}

\section{Diseño}

El diseño del estudio es de tipo exploratorio descriptivo, identifica percepciones, opiniones y comportamientos de los profesionales de la salud durante la epidemia de COVID-19 desde una perspectiva de género. La metodología de investigación es cuantitativa transversal y analiza el fenómeno estudiado durante las semanas centrales de la crisis sanitaria en el ámbito nacional (España). La recogida de información se realizó mediante cuestionario autocompletado. Para su diseño se realizó una revisión bibliográfica en las bases de datos PubMed, Google Scholar y Dialnet sobre la perspectiva de género y se seleccionaron los artículos que tenían relación con el estudio.

\section{Población}

La población objeto de estudio son profesionales del sector salud en activo en hospitales y atención primaria, de centros públicos y privados, durante la crisis de la COVID-19 en España. 


\section{Muestra}

La técnica de muestreo es no probabilística por accesibilidad. El criterio de inclusión son profesionales del sector salud en activo durante la crisis de la COVID-19 en España (hospitales y centros de atención primaria). Profesionales del sector salud incluye personal médico, personal de enfermería, personal técnico de cuidado de enfermería (TCAE), personal técnico, farmacia hospitalaria y personal directivo. La muestra la componen 2.478 profesionales sanitarios de 46 provincias diferentes.

\section{Instrumento y variables}

El instrumento para la recogida de los datos es un cuestionario estructurado. Debido al carácter exploratorio del estudio, se plantean diferentes preguntas de investigación construidas a partir de la escasa bibliografía disponible en el momento del diseño (Tabla 1).

Tabla 1. Temas y preguntas de investigación incluidas en el diseño del cuestionario

\begin{tabular}{|c|c|c|}
\hline Tema & Fuente & $\begin{array}{l}\text { Pregunta de } \\
\text { investigación }\end{array}$ \\
\hline \multicolumn{3}{|c|}{ Aspectos profesionales } \\
\hline $\begin{array}{l}\text { Carga de trabajo } \\
\text { sanitario }\end{array}$ & $\begin{array}{l}\text { Solanas (2020) } \\
\text { Holroyd (2008) } \\
\text { Liu (2020) } \\
\text { Wenham (2020) }\end{array}$ & $\begin{array}{l}\text { ¿Mujeres y hombres han asumido la misma } \\
\text { carga de trabajo asistencial durante la crisis del } \\
\text { coronavirus? }\end{array}$ \\
\hline $\begin{array}{l}\text { Implicación en la } \\
\text { toma de } \\
\text { decisiones }\end{array}$ & UN Women (2020) & $\begin{array}{l}\text { ¿Mujeres y hombres han participado de forma } \\
\text { igualitaria en la toma de decisiones para el } \\
\text { abordaje de la enfermedad? }\end{array}$ \\
\hline \multicolumn{3}{|c|}{ Aspectos relacionados con el hogar y los cuidados } \\
\hline $\begin{array}{l}\text { Reparto de tareas } \\
\text { domésticas y de } \\
\text { cuidados }\end{array}$ & $\begin{array}{l}\text { Solanas (2020) } \\
\text { UN Women (2020) }\end{array}$ & $\begin{array}{l}\text { ¿El impacto del confinamiento familiar es } \\
\text { igual para mujeres y hombres? }\end{array}$ \\
\hline \multicolumn{3}{|c|}{ Aspectos emocionales } \\
\hline $\begin{array}{l}\text { Impacto } \\
\text { emocional }\end{array}$ & $\begin{array}{l}\text { Holroyd (2008) } \\
\text { Liu (2020) }\end{array}$ & $\begin{array}{l}\text { ¿La percepción sobre el riesgo de contagio es } \\
\text { igual para hombres y mujeres? } \\
\text { ¿El impacto emocional de la pandemia es igual } \\
\text { para hombres y mujeres? }\end{array}$ \\
\hline
\end{tabular}




\begin{tabular}{|lll|}
\hline Aspectos sociales & & \\
\hline $\begin{array}{l}\text { Salud sexual y } \\
\text { reproductiva }\end{array}$ & $\begin{array}{l}\text { Solanas (2020) } \\
\text { UN Women (2020) }\end{array}$ & $\begin{array}{l}\text { ¿La percepción del acceso a la anticoncepción } \\
\text { y la atención pre y postparto es igual para } \\
\text { hombres y mujeres sanitarios? }\end{array}$ \\
\hline $\begin{array}{l}\text { Violencia de } \\
\text { género }\end{array}$ & Solanas (2020) & ¿La percepción sobre la violencia de género es \\
& Ruiz-Pérez (2020) & igual para hombres y mujeres sanitarias? \\
\hline $\begin{array}{l}\text { Desequilibrios de } \\
\text { género }\end{array}$ & Solanas (2020) & ¿El impacto social de la pandemia afecta más a \\
& $\begin{array}{l}\text { The Lancet (editorial) } \\
\text { las mujeres? }\end{array}$ \\
& UN Women (2020) & \\
\hline Reconocimiento & - & \\
social & Wenham (2020) & igual para mujeres y hombres? \\
\hline
\end{tabular}

El objetivo que se persigue con el cuestionario es traducir las variables empíricas, sobre las que se desea información, en preguntas concretas capaces de suscitar respuestas fiables, válidas y susceptibles de ser cuantificadas. El cuestionario incluye 30 preguntas divididas en características sociodemográficas, aspectos profesionales, aspectos relacionados con el hogar y los cuidados, aspectos emocionales, aspectos sociales sobre el impacto de la pandemia, con el género como variable determinante. Para la validación de la comprensión del cuestionario, se realizó prueba piloto con 5 profesionales ( 2 medicina, 2 enfermería, 1 auxiliar), que derivó en una modificación en la pregunta referida a la profesión.

\section{Técnica de recogida de datos}

Encuesta administrada por correo electrónico con acceso a cuestionario en línea. Para la captación de participantes se solicitó la colaboración voluntaria de los colegios profesionales médicos y de enfermería, para que distribuyeran el cuestionario entre las personas colegiadas. No se tiene constancia del número de colegios que realizaron difusión del cuestionario. También se solicitó la colaboración a dos sociedades, la Sociedad Española de Calidad Asistencial y la Sociedad Española de Directivos de la salud, que distribuyeron la encuesta entre las personas asociadas previa validación de la junta directiva de ambas. 
La recogida de los datos se realizó mediante el cuestionario estructurado autoadministrado a través de un aplicativo informático en línea. El trabajo de campo tuvo lugar entre los meses de abril y mayo de 2020.

\section{Análisis de datos}

Se realizó un análisis descriptivo de las principales características de las personas participantes según el género. Todas las variables eran categóricas, fueron descritas por medio del número de casos y el porcentaje respecto al total por categoría y según el género. No se tuvieron en cuenta los datos missing. Para evaluar las diferencias de cada variable según el género, se realizó un Test Chi-cuadrado.

La significación estadística se fijó a un nivel de 0,05 (p-valor $<0.05$ ). El paquete estadístico que se utilizó para realizar el tratamiento de los datos y su análisis estadístico fue el R versión 3.5.0., usando la librería «compareGroups».

\section{Consideraciones éticas}

Todos los datos obtenidos en esta investigación han sido tratados de manera anónima para preservar la confidencialidad, conforme a la Ley Orgánica 3/2018, de 5 de diciembre, de protección de datos personales y garantía de los derechos digitales.

El estudio no ha pasado por un CEIC, ni se ha solicitado consentimiento informado a las personas participantes, en tanto que la información recogida es opinión de profesionales mediante un cuestionario de respuesta voluntaria que no incluye datos personales.

\section{RESULTADOS}

\section{Características sociodemográficas}

En primer lugar, se presenta la descripción de los participantes y las características sociodemográficas (Tabla 2). El total de participantes que respondieron a la encuesta fue de 2.478 profesionales de la salud (426 hombres y 2.052 mujeres), de 46 provincias diferentes. La edad de mayor representatividad ha sido de 30 a 50 años (189 hombres y 1.014 mujeres), seguido de más de 50 años (181 hombres y 771 mujeres). 
Tabla 2. Descripción características sociodemográficas de las personas encuestadas

\begin{tabular}{|c|c|c|c|c|}
\hline & Total & \multirow[t]{2}{*}{$\begin{array}{r}\text { Hombre } \\
\mathbf{N}=\mathbf{4 2 6}\end{array}$} & \multirow{2}{*}{$\begin{array}{r}\text { Mujer } \\
\mathbf{N}=\mathbf{2 . 0 5 2}\end{array}$} & \multirow{2}{*}{$\begin{array}{l}\text { Test Chi } \\
\text { p-value }\end{array}$} \\
\hline & $\mathrm{N}=2.478$ & & & \\
\hline \multicolumn{4}{|l|}{ Edad } & 0,127 \\
\hline$[18-30)$ & $293(12,0 \%)$ & $52(12,3 \%)$ & $241(11,9 \%)$ & \\
\hline$[30-50)$ & $1.203(49,1 \%)$ & $189(44,8 \%)$ & $1.014(50,0 \%)$ & \\
\hline$>=50$ & $952(38,9 \%)$ & $181(42,9 \%)$ & $771(38,1 \%)$ & \\
\hline \multicolumn{4}{|l|}{ Profesión } & $<0,0001$ \\
\hline Directivo/a & $125(5,0 \%)$ & $43(10,1 \%)$ & $82(4,0 \%)$ & \\
\hline Enfermero/a & $967(39,0 \%)$ & $109(25,6 \%)$ & $858(41,8 \%)$ & \\
\hline Farmacéutico/a & $19(0,8 \%)$ & $1(0,2 \%)$ & $18(0,9 \%)$ & \\
\hline Médico/a & $549(22,2 \%)$ & $138(32,4 \%)$ & $411(20,0 \%)$ & \\
\hline TCAE & $362(14,6 \%)$ & $29(6,8 \%)$ & $333(16,2 \%)$ & \\
\hline Técnico/a & $151(6,1 \%)$ & $43(10,1 \%)$ & $108(5,3 \%)$ & \\
\hline Otro/a & $305(12,3 \%)$ & $63(14,8 \%)$ & $242(11,8 \%)$ & \\
\hline \multicolumn{4}{|l|}{ Lugar de trabajo } & 0,191 \\
\hline Centro Atención Primaria & $565(22,9 \%)$ & $105(24,7 \%)$ & $460(22,6 \%)$ & \\
\hline $\begin{array}{l}\text { Hospital de la Red de } \\
\text { Utilización Pública }\end{array}$ & $1.594(64,7 \%)$ & $257(60,5 \%)$ & $1.337(65,6 \%)$ & \\
\hline Hospital Privado & $73(3,0 \%)$ & $14(3,3 \%)$ & $59(2,9 \%)$ & \\
\hline Otros & $232(9,4 \%)$ & $49(11,5 \%)$ & $183(9,0 \%)$ & \\
\hline \multicolumn{4}{|l|}{ Estado civil } & 0,2252 \\
\hline Casado/a & $1.344(54,3 \%)$ & $232(54,6 \%)$ & $1.112(54,2 \%)$ & \\
\hline Divorciado/a & $184(7,4 \%)$ & $24(5,6 \%)$ & $160(7,8 \%)$ & \\
\hline En pareja & $448(18,1 \%)$ & $68(16,0 \%)$ & $380(18,5 \%)$ & \\
\hline Separado/a & $51(2,1 \%)$ & $9(2,1 \%)$ & $42(2,0 \%)$ & \\
\hline Soltero/a & $423(17,1 \%)$ & $87(20,5 \%)$ & $336(16,4 \%)$ & \\
\hline Viudo/a & $27(1,1 \%)$ & $5(1,2 \%)$ & $22(1,1 \%)$ & \\
\hline \multicolumn{4}{|l|}{ Personas a tu cargo } & 0,048 \\
\hline Ninguna & $957(38,9 \%)$ & $186(43,9 \%)$ & $771(37,8 \%)$ & \\
\hline Solo hijos & $1.302(52,9 \%)$ & $213(50,2 \%)$ & $1.089(53,5 \%)$ & \\
\hline Solo padres & $96(3,9 \%)$ & $14(3,3 \%)$ & $82(4,0 \%)$ & \\
\hline Hijos y padres & $106(4,3 \%)$ & $11(2,6 \%)$ & $95(4,7 \%)$ & \\
\hline \multicolumn{4}{|c|}{ Lugar de residencia habitual durante la pandemia } & 0,333 \\
\hline Hotel & $21(0,8 \%)$ & $6(1,4 \%)$ & $15(0,7 \%)$ & \\
\hline Piso compartido & $109(4,4 \%)$ & $24(5,6 \%)$ & $85(4,2 \%)$ & \\
\hline Vivienda de otro familiar & $133(5,4 \%)$ & $25(5,9 \%)$ & $108(5,3 \%)$ & \\
\hline Vivienda propia & $2.144(86,8 \%)$ & $359(84,5 \%)$ & $1.785(87,2 \%)$ & \\
\hline Otros & $64(2,6 \%)$ & $11(2,6 \%)$ & $53(2,6 \%)$ & \\
\hline
\end{tabular}

En cuando a la profesión, hay representatividad de diferentes disciplinas. Entre los hombres participantes $(n=426)$, el $32,4 \%$ son médicos, el 25,6\% enfermeros, el 10,1\% técnicos, el 10,1\% directivos, el 6,8\% TCAE y el 15,0\% otros. Las mujeres participantes 
$(n=2.052)$ se distribuyen en un 41,8\% enfermeras, un 20,0\% médicas, un 16,2\% TCAE, un 5,3\% técnicas, un $4 \%$ directivas y el $12,7 \%$ otras. La mayoría de los profesionales que contestan el cuestionario, el 64,7\% (257 hombres y 1.337 mujeres) trabajan en hospitales públicos, el 22,9\% (105 hombres y 460 mujeres) trabajan en atención primaria y solo un $3 \%$ (14 hombres y 59 mujeres) en hospital privado.

El 54,3\% (232 hombres y 1.112 mujeres) está casado/a, el 18,1\% (68 hombres y 380 mujeres) vive en pareja y solo el $27,7 \%$ (125 hombres y 560 mujeres) son personas sin pareja, solteras, divorciadas, separadas o viudas. Más de la mitad, el 52,9\% (213 hombres y 1.089 mujeres) tienen hijos/as a su cargo, el 4,3\% (11 hombres y 95 mujeres) hijos/as y padres y el 3,9\% (14 hombres y 82 mujeres) refieren tener a los padres. El 38,9\% (186 hombres y 771 mujeres) no tiene personas a cargo.

La residencia habitual para el 86,8\% (359 hombres y 1.785 mujeres) ha sido la vivienda propia. El 5,4\% (25 hombres y 108 mujeres) han estado en vivienda familiar, el $4,4 \%$ en pisos compartidos ( 24 hombres y 85 mujeres) y el 3,4\% en hotel u otros (17 hombres y 68 mujeres).

\section{Aspectos profesionales}

En cuanto a los aspectos relacionados con la actividad profesional de las personas encuestadas (Tabla 3), a la pregunta cuantas horas han trabajado la última semana, el $52,0 \%(\mathrm{n}=219)$ de hombres y el 59,4\% $(\mathrm{n}=1.182)$ de mujeres han realizado jornada completa (21-40 horas), el 43,2\% $(n=182)$ de hombres y el 34,2\% $(n=680)$ de mujeres refiere haber trabajado más horas de la jornada ( $\mathrm{p}$-valor $=0,005)$.

En referencia a las horas en contacto directo con pacientes la última semana, el 28,4\% $(\mathrm{n}=118)$ de los hombres y el 27,2\% $(\mathrm{n}=541)$ de las mujeres contestan de once a treinta horas, y el 29,8\% $(\mathrm{n}=124)$ de los hombres y el 36,3\% $(\mathrm{n}=722)$ de las mujeres responden que prácticamente han estado en contacto todas las horas ( $\mathrm{p}$-valor $=0,072)$.

El 57,8\% ( $\mathrm{n}=245)$ de los hombres no ha realizado teletrabajo la última semana y el $16,3 \%(n=69)$ ha hecho diez horas o menos. En el caso de las mujeres, han sido el 66,8\% $(n=1.342)$ y el $12,3 \%(n=247)$, respectivamente $(p$-valor $=0,005)$.

A la pregunta si han formado parte de alguna comisión o grupo de trabajo durante la pandemia, el 61,2\% $(n=259)$ de los hombres y el 74,5\% (1.505) de las mujeres refiere que no (p-valor $<0,001)$. Y sobre si se ha tenido en cuenta su opinión a la hora de tomar 
decisiones, siempre y casi siempre contestan el 27,1\% $(n=115)$ de los hombres y el 19,2\% $(n=392)$ de las mujeres. Y nunca o rara vez el 37,2\% $(n=158)$ de los hombres y el 42,1\% $(\mathrm{n}=860)$ de las mujeres ( $\mathrm{p}$-valor=0,015).

Tabla 3. Descripción aspectos profesionales de las personas encuestadas

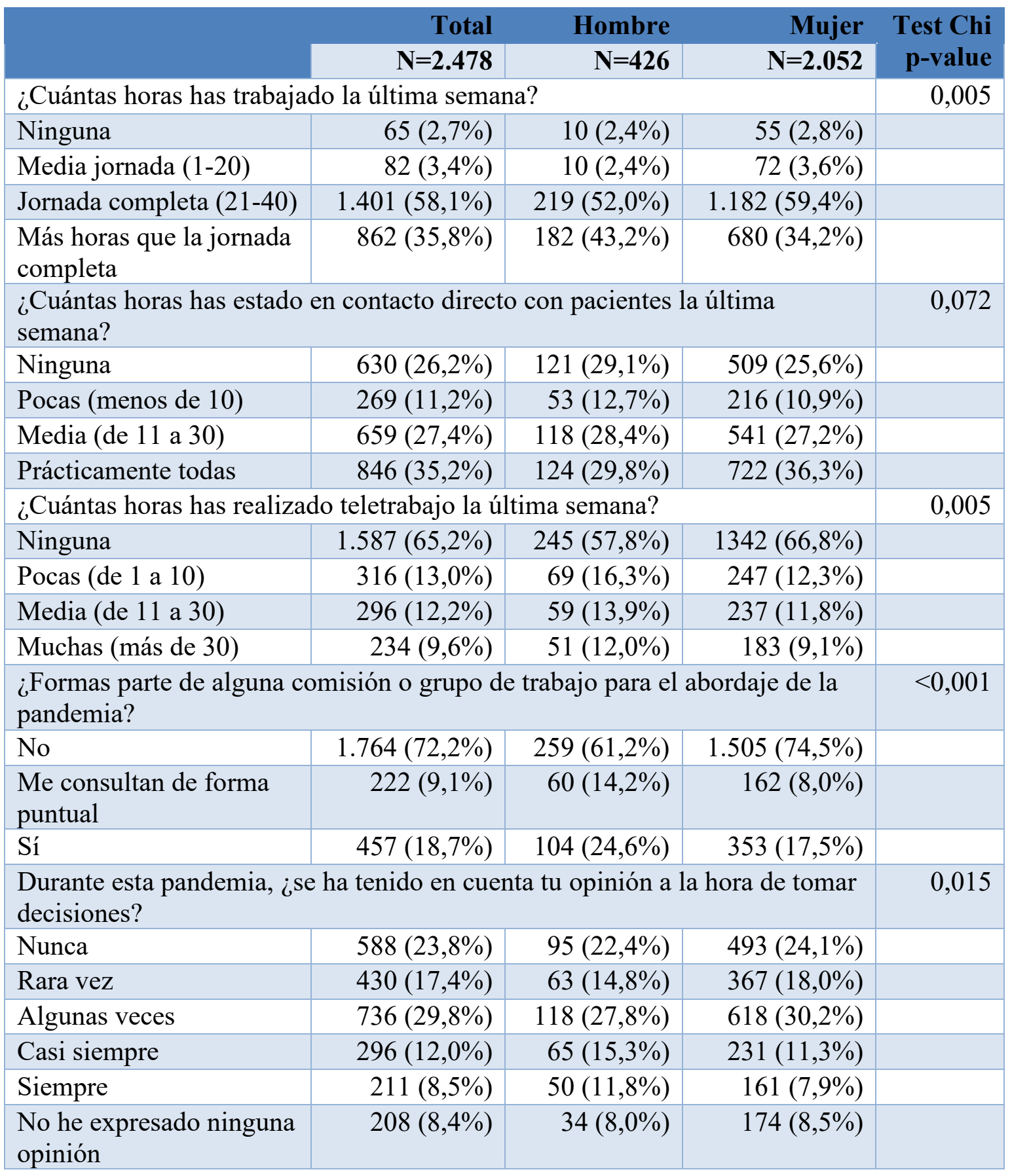

\section{Aspectos relacionados con el hogar y los cuidados}

En relación con las tareas del hogar y los cuidados (Tabla 4), el 65,1\% de las mujeres $(n=1.298)$, contestan que han dedicado a tareas del hogar de 1 a 14 horas la última semana, 
y el 32,7\% (n=652) más de 25 horas. El 67,1\% de los hombres ha dedicado de 1 a 14 horas y el 30,3\% más de 25 horas. No se observan diferencias significativas en la dedicación a las tareas del hogar ( $\mathrm{p}$-valor $=0,590)$.

Tabla 4. Descripción aspectos relacionados con el hogar y los cuidados

\begin{tabular}{|c|c|c|c|c|}
\hline & Total & Hombre & Mujer & Test Chi \\
\hline & $\mathrm{N}=\mathbf{2 . 4 7 8}$ & $\mathrm{N}=426$ & $\mathrm{~N}=\mathbf{2 . 0 5 2}$ & \\
\hline ¿Cuántas horas has dedic & la última seman & a tareas del hc & gar? & 0,590 \\
\hline Ninguna & $55(2,3 \%)$ & $11(2,6 \%)$ & $44(2,2 \%)$ & \\
\hline De 1 a 14 & $1.581(65,4 \%)$ & $283(67,1 \%)$ & $1.298(65,1 \%)$ & \\
\hline Más de 25 & $780(32,3 \%)$ & $128(30,3 \%)$ & $652(32,7 \%)$ & \\
\hline ¿Cuántas horas has dedic & la última seman & a cuidado de 1 & jos/as? & $<0,001$ \\
\hline No tengo hijos & $1.461(59,8 \%)$ & $273(64,2 \%)$ & $1.188(58,9 \%)$ & \\
\hline Ninguna & $43(1,8 \%)$ & $9(2,1 \%)$ & $34(1,7 \%)$ & \\
\hline De 1 a 10 & $206(8,4 \%)$ & $33(7,8 \%)$ & $173(8,6 \%)$ & \\
\hline De 11 a 20 & $168(6,9 \%)$ & $44(10,4 \%)$ & $124(6,1 \%)$ & \\
\hline De 21 a 56 & $318(13,0 \%)$ & $49(11,5 \%)$ & $269(13,3 \%)$ & \\
\hline $\begin{array}{l}\text { Todas las que no estoy } \\
\text { trabajando }\end{array}$ & $247(10,1 \%)$ & $17(4,0 \%)$ & $230(11,4 \%)$ & \\
\hline $\begin{array}{l}\text { ¿Cuántas horas has dedic } \\
\text { dependientes? }\end{array}$ & a última seman & a cuidado de & rsonas & 0,015 \\
\hline $\begin{array}{l}\text { No tengo personas a mi } \\
\text { cargo }\end{array}$ & $1.939(85,3 \%)$ & $361(90,2 \%)$ & $1.578(84,2 \%)$ & \\
\hline Ninguna & $9(0,4 \%)$ & $0(0,0 \%)$ & $9(0,5 \%)$ & \\
\hline De 1 a 10 & $216(9,5 \%)$ & $30(7,5 \%)$ & $186(9,9 \%)$ & \\
\hline 21 o más & $88(3,9 \%)$ & $7(1,8 \%)$ & $81(4,3 \%)$ & \\
\hline $\begin{array}{l}\text { Todo el tiempo que está } \\
\text { en casa }\end{array}$ & $22(1,0 \%)$ & $2(0,5 \%)$ & $20(1,1 \%)$ & \\
\hline $\begin{array}{l}\text { ¿Has sentido dificultad p } \\
\text { pandemia? }\end{array}$ & mpaginar tu $\mathrm{v}$ & familiar y 1 & ral durante la & 0,225 \\
\hline Nunca o rara vez & $874(35,4 \%)$ & $164(38,5 \%)$ & $710(34,7 \%)$ & \\
\hline Algunas veces & $836(33,8 \%)$ & $144(33,8 \%)$ & $692(33,9 \%)$ & \\
\hline Siempre o casi siempre & $760(30,8 \%)$ & $118(27,7 \%)$ & $642(31,4 \%)$ & \\
\hline
\end{tabular}

A la pregunta cuantas horas ha dedicado la última semana al cuidado de sus hijos, el $8,6 \%(n=173)$ de las mujeres contestan de 1 a 10 horas, el 6,1\% $(n=124)$ de 11 a 20 y el 13,3\% (n=269) de 21 a 56 horas. En el caso de los hombres, la distribución es del 7,8\% $(\mathrm{n}=33), 10,4 \%(\mathrm{n}=44)$ y $11,5 \%(\mathrm{n}=49)$, respectivamente. El 11,4\% $(\mathrm{n}=230)$ de las mujeres y el 4,0\% $(n=17)$ de los hombres refieren dedicar todas las que no están trabajando (pvalor $<0,001)$. Sobre el cuidado de personas dependientes, el 15,3\% ( $\mathrm{n}=287)$ de las mujeres y el 9,8\% $(n=39)$ de los hombres les ha dedicado alguna hora la última semana (p-valor $=0,015)$. 
Si ha sentido dificultad para compaginar su vida familiar y laboral durante la pandemia, contestaron siempre o casi siempre el $31,4 \%(n=642)$ de las mujeres y el $27,7 \%$ $(n=118)$ de los hombres, nunca o rara vez el 34,7\% $(n=710)$ y el 38,5\% $(n=164)$, respectivamente ( $\mathrm{p}$-valor $=0,225)$.

\section{Aspectos emocionales}

En cuanto a los aspectos emocionales (Tabla 5), a la pregunta si han sentido ansiedad durante la pandemia, el 32,0\% $(\mathrm{n}=654)$ de las mujeres contestaron siempre o casi siempre y el 48,6\% ( $n=993)$ algunas veces. Los valores son inferiores en el caso de los hombres: el 18,6\% (n=79) sintió ansiedad siempre o casi siempre y el 47,3\% $(n=201)$ algunas veces (p-valor $<0,001)$.

Si ha tenido dificultad para concentrarse, el 25,6\% $(n=521)$ de las mujeres refieren siempre o casi siempre y el 53,1\% $(n=1.083)$ algunas veces. El 17,5\% $(n=74)$ de los hombres ha tenido dificultad para concentrarse siempre o casi siempre y el 48,2\% $(n=204)$ algunas veces ( $\mathrm{p}$-valor $<0,001)$.

Referente a la pregunta si ha tenido dificultades para dormir, el 39,2\% $(\mathrm{n}=801)$ de las mujeres dicen siempre o casi siempre y algunas veces el 42,2\% $(n=862)$. En el caso de los hombres, han tenido dificultad para dormir siempre o casi siempre el 29,0\% ( $n=123)$ $y$ algunas veces el 38,7\% (n=164) (p-valor $<0,001)$.

Si ha sentido miedo a contagiarse de la COVID-19, el 38,2\% $(n=781)$ de las mujeres han contestado siempre o casi siempre y el 39,1\% $(n=800)$ algunas veces. En los hombres, estos valores han sido el $31,2 \%(n=133)$ y el $34,3 \%(n=146)$, respectivamente (pvalor $<0,001)$. 
Tabla 5. Descripción aspectos emocionales

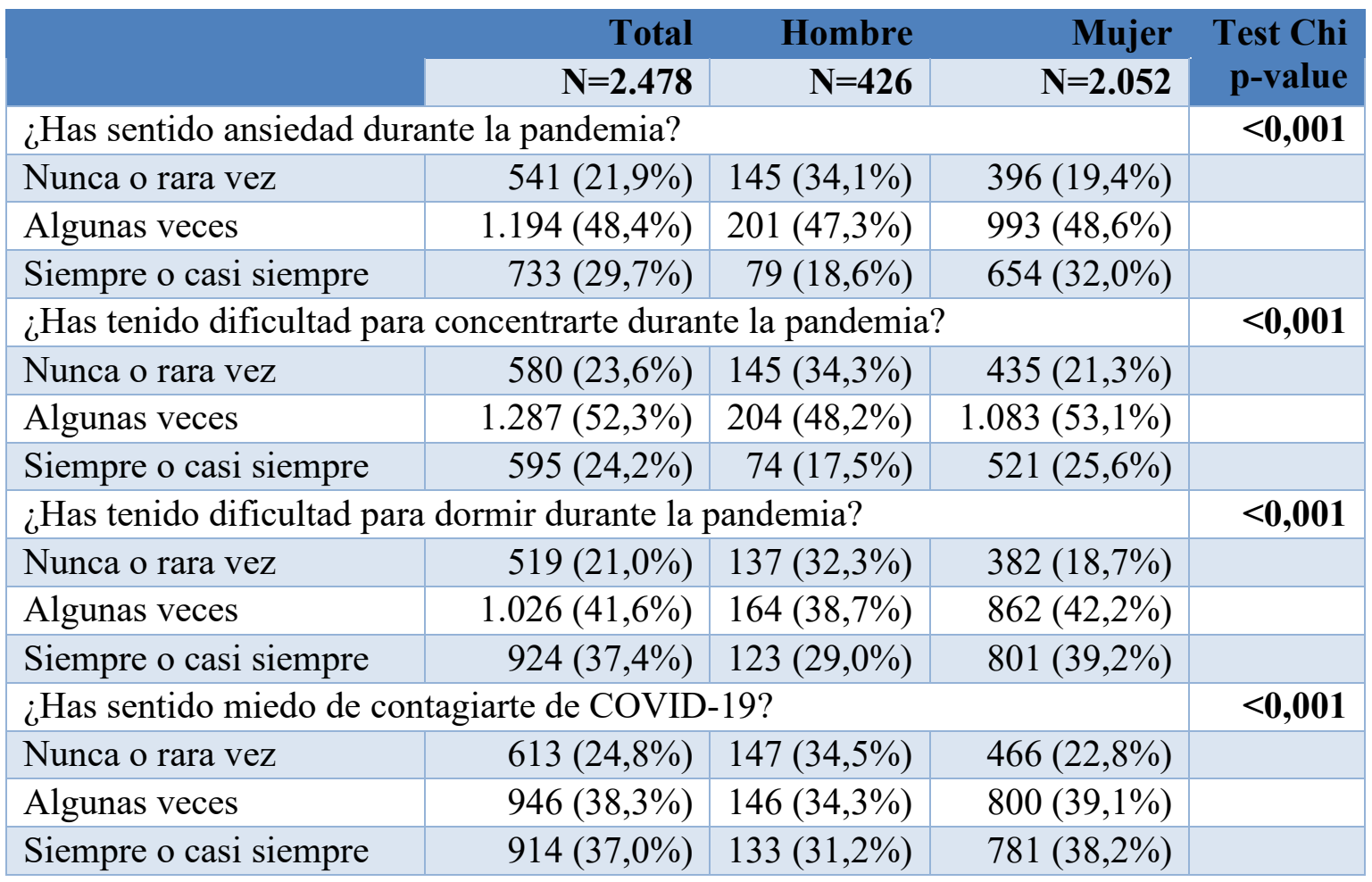

\section{Aspectos sociales}

Respecto a la opinión de las personas encuestadas sobre el impacto social de la COVID19 (Tabla 6), a la pregunta si creen que la enfermedad está teniendo un impacto diferente en hombres y mujeres, el 46,2\% $(n=945)$ de las mujeres y el 40,0\% $(n=170)$ de los hombres opinan que sí. Solo un 19,0\% $(n=389)$ de las mujeres y un $28,2 \%(n=210)$ de los hombres considera que no ha habido impacto diferenciado según el género ( $p$ valor $<0,001)$. Referente a la pregunta si esta crisis afectará más a las mujeres, el 39,6\% de las mujeres contestan que sí, frente al 19,0\% $(n=81)$ de los hombres. Un 23,5\% $(n=482)$ de las mujeres considera que no, frente al 40,6\% $(n=173)$ de los hombres que opina lo mismo (p-valor $<0,001)$.

Si consideran que la mujer ha visto disminuido el acceso a la anticoncepción y a la atención pre y postparto, no hay diferencias significativas entre hombres y mujeres. El $39,8 \%$ ( $n=814)$ de las mujeres y el 35,95 de los hombres considera que sí, y el 17,4\% $(n=355)$ de las mujeres y el 21,7\% $(n=92)$ de los hombres afirma que no ( $p$-valor $=0,079)$.

El 90,1\% ( $\mathrm{n}=1.843)$ de las mujeres y el $80,6 \%(\mathrm{n}=341)$ de los hombres consideran que hay más riesgo de violencia de genero con el confinamiento. Solo un $1,5 \%(n=31)$ de las mujeres y un $4,3 \%(n=18)$ de los hombres dice que no ( $p$-valor $<0,001)$. Respecto a la valoración del reconocimiento social, el 54,0\% $(\mathrm{n}=119)$ de las mujeres y el 53,3\% 
$(\mathrm{n}=226)$ de los hombres sienten que la ciudadanía está reconociendo su trabajo durante la pandemia. E1 37,3\% $(n=766)$ de las mujeres y el 34,2\% $(n=145)$ de los hombres ha sentido el reconocimiento algunas veces (p-valor=0,009). El 31,8\% $(n=653)$ de las mujeres cree que la reputación de la profesión habrá mejorado cuando finalice la pandemia, y un 42,4\% $(\mathrm{n}=871)$ considera que no. No existen diferencias significativas respecto a sus compañeros de profesión, un $37,1 \%(n=158)$ cree que sí mejorará y un $41,5 \%(n=177)$ afirma que no ( $\mathrm{p}$-valor $=0,056)$.

Tabla 6. Descripción aspectos sociales

\begin{tabular}{|c|c|c|c|c|}
\hline & $\begin{array}{r}\text { Total } \\
\mathrm{N}=2.478\end{array}$ & $\begin{array}{r}\text { Hombre } \\
\text { N=426 }\end{array}$ & $\begin{array}{r}\text { Mujer } \\
\mathbf{N}=2.052\end{array}$ & $\begin{array}{l}\text { Test Chi } \\
\text { p-value }\end{array}$ \\
\hline $\begin{array}{l}\text { ¿Crees que la e } \\
\text { mujeres? }\end{array}$ & niendo un impact & diferente en h & bres que en & $<0,001$ \\
\hline No & $509(20,6 \%)$ & $120(28,2 \%)$ & $389(19,0 \%)$ & \\
\hline No lo sé & $845(34,2 \%)$ & $135(31,8 \%)$ & $710(34,7 \%)$ & \\
\hline Sí & $1.115(45,2 \%)$ & $170(40,0 \%)$ & $945(46,2 \%)$ & \\
\hline ¿Crees que esta & s a las mujeres? & & & $<0,001$ \\
\hline No & $655(26,5 \%)$ & $173(40,6 \%)$ & $482(23,5 \%)$ & \\
\hline No lo sé & $929(37,5 \%)$ & $172(40,4 \%)$ & $757(36,9 \%)$ & \\
\hline Sí & $892(36,0 \%)$ & $81(19,0 \%)$ & $811(39,6 \%)$ & \\
\hline $\begin{array}{l}\text { ¿Consideras qu } \\
\text { la atención pre }\end{array}$ & $\begin{array}{l}\text { visto disminuido } \\
\text { e la pandemia? }\end{array}$ & 1 acceso a la ar & oncepción y a & 0,079 \\
\hline No & $447(18,1 \%)$ & $92(21,7 \%)$ & $355(17,4 \%)$ & \\
\hline No lo sé & $1.054(42,7 \%)$ & $179(42,3 \%)$ & $875(42,8 \%)$ & \\
\hline Sí & $966(39,2 \%)$ & $152(35,9 \%)$ & $814(39,8 \%)$ & \\
\hline $\begin{array}{l}\text { ¿Consideras qu } \\
\text { riesgo de violer }\end{array}$ & de la tensión deb & do al confinam & to, hay más & $<0,001$ \\
\hline No & $49(2,0 \%)$ & $18(4,3 \%)$ & $31(1,5 \%)$ & \\
\hline No lo sé & $236(9,6 \%)$ & $64(15,1 \%)$ & $172(8,4 \%)$ & \\
\hline Sí & $2.184(88,5 \%)$ & $341(80,6 \%)$ & $1.843(90,1 \%)$ & \\
\hline Durante la panc & e la ciudadanía e & á reconociendo & trabajo? & 0,009 \\
\hline Nunca & $56(2,3 \%)$ & $18(4,2 \%)$ & $38(1,9 \%)$ & \\
\hline Rara vez & $173(7,0 \%)$ & $35(8,3 \%)$ & $138(6,7 \%)$ & \\
\hline Algunas veces & $911(36,8 \%)$ & $145(34,2 \%)$ & $766(37,3 \%)$ & \\
\hline Casi siempre & $1.007(40,7 \%)$ & $161(38,0 \%)$ & $846(41,2 \%)$ & \\
\hline Siempre & $328(13,3 \%)$ & $65(15,3 \%)$ & $263(12,8 \%)$ & \\
\hline $\begin{array}{l}\text { Cuando finalice } \\
\text { mejorado respe }\end{array}$ & $\begin{array}{l}\text { es que la reputa } \\
\text { andemia? }\end{array}$ & ón de tu profes & habrá & 0,056 \\
\hline No & $1.048(42,3 \%)$ & $177(41,5 \%)$ & $871(42,4 \%)$ & \\
\hline No lo sé & $619(25,0 \%)$ & $91(21,4 \%)$ & $528(25,7 \%)$ & \\
\hline Sí & $811(32,7 \%)$ & $158(37,1 \%)$ & $653(31,8 \%)$ & \\
\hline
\end{tabular}




\section{DISCUSIÓN}

Como ha sucedido en otras pandemias (véase, por ejemplo, Holroyd et al., 2008; Wenham et al., 2020), la crisis sanitaria de la COVID-19 ha afectado a las mujeres de manera relevante, especialmente en el caso de las profesiones sanitarias, mayoritariamente feminizadas. Durante el período de confinamiento decretado por la alerta sanitaria en España, las mujeres participantes en este estudio han realizado más trabajo presencial y en contacto directo con pacientes que los hombres. Las mujeres han estado en la primera línea de atención al paciente durante la pandemia, asumiendo mayor carga de trabajo sanitario, como apunta Solanas (2020).

Asimismo, un elevado número de profesionales de la salud declara haber trabajado más horas de la jornada laboral habitual. No obstante, en este caso, fue mayor el porcentaje en los hombres, un $43,2 \%$ frente al $34,2 \%$ de las profesionales sanitarias. El mayor número de horas en el caso de los hombres podría explicarse porque se encuentran en puestos más flexibles para la realización de teletrabajo, como técnicos y directivos, que estudios preliminares (véase González, 2020) asocian con un incremento de la jornada laboral.

Por otra parte, la pandemia ha evidenciado desigualdades que ya existen en referencia a la toma de decisiones. Sobre la participación en la gestión de la pandemia, solo un 17,5\% de mujeres ha formado parte de comisiones o grupos de trabajo y un $8 \%$ ha sido consultada de forma puntual, frente al 24,6\% y 14,2\% de los hombres. En general, un elevado número de profesionales considera que no se tiene en cuenta su opinión, pero de nuevo el porcentaje es más elevado entre las mujeres, un $42,1 \%$ de mujeres frente al $37,2 \%$ de hombres. Estas observaciones coinciden con las constatadas por CastellanosTorres (2020), que afirma que hay una escasa participación de mujeres en la toma de decisiones, pese a ser el colectivo mayoritario dentro del sector sanitario.

Posenato (2020) también ha observado una menor dedicación de las mujeres al desarrollo de tareas de investigación, debido al aumento del tiempo dedicado a actividades domésticas y de cuidado durante el confinamiento familiar. En España, un $84 \%$ de las mujeres realiza tareas del hogar y un 95\% asume el cuidado y educación de hijos e hijas diariamente según recogen los datos de Eurostat (2017). Esta situación afecta de manera más acusada a las familias monoparentales, un $82 \%$ de las cuales están encabezadas por mujeres como recoge la Fundación Adecco (2019). Durante el 
confinamiento, algunos estudios (véase Alon et al., 2020) han observado una tendencia al reparto más igualitario en dedicación a las tareas del hogar, aunque las tareas de cuidado siguen recayendo mayoritariamente en las mujeres, como observan Farré et al. (2020), especialmente el cuidado de los menores por el cierre de los colegios.

En este sentido, respecto al impacto del confinamiento familiar en profesionales de la salud participantes en el estudio, la dedicación de hombres y mujeres a tareas del hogar ha sido muy similar. Sobre la conciliación de la vida laboral y familiar, hombres y mujeres lo perciben de forma similar, más del $60 \%$ ha sentido dificultades algunas veces, casi siempre o siempre. A pesar de esta percepción, en cuanto al cuidado de hijos e hijas, las mujeres dedican más tiempo, siendo especialmente significativo que un 11,4\% de mujeres dice dedicarles todo el tiempo en el cual no están trabajando, frente a un $4 \%$ de hombres que afirma lo mismo. Las mujeres también dedican más tiempo al cuidado de personas dependientes, un $15,3 \%$ ha dedicado alguna hora a la semana, frente al 9,8\% de los hombres. Ellas han asumido la mayor parte de la responsabilidad de cuidados en la familia.

Además, el miedo al contagio por parte de las profesionales de la salud ha sido especialmente alto durante la pandemia de COVID-19, un 77,3\% frente al 65,5\% de sus compañeros de profesión. Ellas han tenido un contacto más cercano con los pacientes, además de suplir responsabilidades de atención básica que habitualmente asumen familiares y cuidadores, que debido a la política de aislamiento no podían estar en las dependencias de los centros sanitarios. Estos factores parecen determinantes en el impacto emocional que generan (véase Liu, 2020). Las mujeres han sentido mayor ansiedad, dificultad para concentrarse y para dormir.

Al preguntar a las personas encuestadas sobre las consecuencias de la pandemia en aspectos sociales con impacto de género, un alto porcentaje considera que tendrá un impacto diferente en hombres y en mujeres, pero son las mujeres quiénes consideran que la crisis las afectará más a ellas, un 39,6\% así lo afirma frente al 19\% de hombres. Entre los principales impactos percibidos por el 39,8\% de mujeres y el 35,9\% de hombres, se encuentra la disminución del acceso a la anticoncepción y la atención al preparto y postparto durante la pandemia.

Especialmente destacable es que un $90,1 \%$ de mujeres y $80,6 \%$ considera que hay más riesgos de violencia de género durante el confinamiento. En España, el estado de 
alarma y el confinamiento han provocado un incremento de la violencia contra la mujer como recoge el Instituto de la Mujer y para la Igualdad de Oportunidades (2020). La violencia de género es un tema sobre el que existe una amplia evidencia documentada (véase, por ejemplo, Usher et al., 2020 y Ruiz-Pérez et al., 2020), que se agrava en situaciones de emergencia, como manifiesta la Organización de Naciones Unidas (ONU Mujeres, 2020).

Por último, hay que destacar que las profesionales de la salud se sienten más reconocidas por la ciudadanía que sus compañeros, un 91,3\% de las mujeres han sentido algunas veces, siempre o casi siempre este reconocimiento frente al el 87,5\% de los hombres. Podría ser porque mayoritariamente ellas ocupan puestos que están en relación directa con el paciente, como enfermería, TCAE y médicas. No obstante, el 42,4\% considera que cuando finalice la pandemia la reputación de su profesión no habrá mejorado.

Como limitaciones de este estudio se encuentra la representatividad de la muestra, derivada de la autoselección de participantes, que no refleja fielmente la población de profesionales del sector salud. Asimismo, al tratarse de un estudio con carácter exploratorio, se abordan diversos temas a través de los cuales se pretenden identificar desigualdades, pero sería interesante en futuras investigaciones ahondar en profundidad en cada uno de los aspectos tratados.

\section{CONCLUSIONES}

Como ha sucedido en otras pandemias, el impacto de género ha sido ignorado en la crisis de la COVID-19. Sin embargo, esta crisis afecta a las mujeres de manera muy relevante, por ejemplo en los cuidados, que recaen mayoritariamente en las mujeres, especialmente el cuidado de los menores por el cierre de los colegios. En este estudio se ha observado que las profesionales de la salud asumen una mayor carga de cuidado que sus compañeros.

La pandemia también ha evidenciado temas importantes sobre desigualdades que ya existen en referencia a la toma de decisiones, que se recogen en este estudio, exponiendo las vulnerabilidades del sistema y la necesidad de realizar un abordaje con perspectiva de género ante las pandemias. La diferente percepción de hombres y mujeres sobre algunos de los aspectos relacionados con la salud, como la violencia de género y el impacto social de la enfermedad, deben tenerse en cuenta por su repercusión en la toma decisiones. 
El papel de la mujer debe ser tenido en cuenta en el abordaje de la crisis por la COVID-19 y debe integrarse en las decisiones tal y como indican organismos internacionales como la Organización de Naciones Unidas. Especialmente debe implicarse a las mujeres en la toma de decisiones en el ámbito de la salud, para tratar aspectos relacionados con la gestión de las pandemias, así como las consecuencias sociales y emocionales que afectan a las profesionales derivadas de la carga asistencial y la falta de corresponsabilidad en los cuidados. 


\section{BIBLIOGRAFÍA}

ALON T., et al. The Impact of COVID-19 on Gender Equality. En: NBER Working Paper. 2020, núm. 26947.

BALlUERKA, N., et al. Las consecuencias psicológicas de la COVID-19 y el confinamiento. Servicio de Publicaciones de la Universidad del País Vasco: 2020. [Consulta: 20 de mayo de 2020]. Disponible en: https://bit.ly/3FEmw9o.

Benlloch, C.; Aguado, E. Teletrabajo y conciliación: el estrés se ceba con las mujeres.En: The Conversation. 29 de abril de 2020. [Consulta: 20 de mayo de 2020]. Disponible en: https://bit.ly/3FFFVGV.

Castellanos-Torres, E.; Mateos J.; Chilet-Rosell, E. COVID-19 en clave de género. En: Gaceta Sanitaria. 2021, vol. 34, pp. 419-421.

EuRostAT. La vida de las mujeres y los hombres en Europa - un retrato estadístico. Unión Europea: 2017. [Consulta: 25 de junio de 2020]. Disponible en: https://www.ine.es/prodyser/myhue17/index.html?lang=es.

FARRÉ, L.; GonZÁLEZ, L. ¿Quién se encarga de las tareas domésticas durante el confinamiento? Covid-19, mercado de trabajo y uso del tiempo en el hogar. En: Nada es gratis. 23 de abril de 2020. [Consulta: 8 de junio de 2020]. Disponible en: https://nadaesgratis.es/admin/quien-se-encarga-de-las-tareas-domesticas.

FUNDACIÓN ADECCO. Informe Monomarentalidad y empleo. Informes Fundación Adecco, Octubre de 2019. [Consulta: 20 de mayo de 2020]. Disponible en: https://bit.ly/3FzaQ84.

GONZÁLEZ, B. El teletrabajo en confinamiento suma dos horas más a la jornada laboral. En: UOC - News. 13 de mayo de 2020. [Consulta: 14 de mayo de 2020]. Disponible en: https://bit.ly/3GIQN8E.

Holroyd, E.; MCNAught, C. The SARS crisis: reflections of Hong Kong nurses. En: International Nursing Review. 2008, vol. 55, núm. 1, pp. 27-33.

INSTITUTO DE LA MUJER Y PARA LA IGUALDAD DE OPORTUNIDADES. La perspectiva de género, esencial en la respuesta a la COVID-19. Instituto de la Mujer, 8 de mayo de 2020. [Consulta: 20 de mayo de 2020]. Disponible en: https://bit.ly/3KkPD5m.

Instituto DE LA MuJER Y PARA LA IGUALDAD DE OPORTUNIDAdes. Tabla Mujeres en Cifras: Profesionales Sanitarios. Instituto de la Mujer: 26 de mayo de 2020. [Consulta: 8 de junio de 2020]. Disponible en: https://bit.ly/3nABAil.

LIU, Q., et al. The experiences of health-care providers during the COVID-19 crisis in China: a qualitative study. En: Lancet Glob Health. 2020, vol. 8, pp. 790-798.

ONU Mujeres. Dimensiones de Género en la crisis del COVID-19 en Colombia. ONU Mujeres Colombia, 2020. [Consulta: 8 de junio de 2020]. Disponible en: https://bit.ly/3KbEq6W. 
Posenato-Garcia, L. Dimensões de sexo, gênero e raça na pesquisa sobre COVID-19. En: Epidemiologia e Serviços de Saúde. 2020, vol. 29, núm. 3, p. e20202207.

Ruiz-PÉrez, I.; PAstor-Moreno, G. Medidas de contención de la violencia de género durante la pandemia de COVID-19. En: Gaceta Sanitaria. 2022, vol. 35, pp. 389394.

SolANAS, M. La crisis del COVID-19 y sus impactos en la igualdad de género. En: Real Instituto Elcano. 1 de abril de 2020. [Consulta: 22 de abril de 2020]. Disponible en: https://bit.ly/3FyVWyn.

THE LANCET [editorial]. The gendered dimensions of COVID-19. En: The Lancet. 2020, vol. 395, p. 1168.

UN Women. Policy Brief: The Impact of COVID-19 on Women. UN Women, 9 de abril de 2020. [Consulta: 22 de abril de 2020]. Disponible en: https://bit.ly/3Ki7lki.

UsHER, K., et al. Family violence and COVID-19: increased vulnerability and reduced options for support. En: International Journal of Mental Health Nursing. 2020, vol. 29, pp. 549-552.

Wenham, C.; SMith, J.; MoRgan, R. COVID-19: the gendered impacts of the outbreak. En: The Lancet. 2020, vol. 395, pp. 846-848. 